\title{
Auditory-vocal coupling in the naked mole-rat, a mammal with poor auditory thresholds
}

\author{
Kazuo Okanoya ${ }^{1,2}$. Shigeto Yosida ${ }^{2}$. Catherine M. Barone ${ }^{3}$. Daniel T. Applegate ${ }^{3}$ Elizabeth F. Brittan-Powell . $^{4}$ \\ Robert J. Dooling ${ }^{4}$ Thomas J. Park ${ }^{3}$ (1)
}

Received: 7 May 2018 / Revised: 4 September 2018 / Accepted: 7 September 2018 / Published online: 19 September 2018

(c) The Author(s) 2018

\begin{abstract}
Naked mole-rats are extremely social and extremely vocal rodents, displaying a wide range of functionally distinct call types and vocalizing almost continuously. Their vocalizations are low frequency, and a behavioral audiogram has shown that naked mole-rats, like other subterranean mammals, hear only low frequencies. Hence, the frequency range of their hearing and vocalizations appears to be well matched. However, even at low frequencies, naked mole-rats show very poor auditory thresholds, suggesting vocal communication may be effective only over short distances. However, in a tunnel environment where low frequency sounds propagate well and background noise is low, it may be that vocalizations travel considerable distances at suprathreshold intensities. Here, we confirmed hearing sensitivity using the auditory brainstem response; we characterized signature and alarm calls in intensity and frequency domains and we measured the effects of propagation through tubes with the diameter of naked mole-rat tunnels. Signature calls—used for intimate communication—could travel 3-8 $\mathrm{m}$ at suprathreshold intensities, and alarm calls (lower frequency and higher intensity), could travel up to $15 \mathrm{~m}$. Despite this species' poor hearing sensitivity, the naked mole-rat displays a functional, coupled auditory-vocal communication system—a hallmark principle of acoustic communication systems across taxa.
\end{abstract}

Keywords Auditory brainstem response $\cdot$ Vocal communication $\cdot$ Auditory threshold $\cdot$ Alarm call $\cdot$ Signature call

\section{Introduction}

Naked mole-rats are extremely social rodents. They live in large colonies that can include hundreds of individuals, with usually only one breeding female and one to three breeding males. The remaining adults are divided into at least two non-breeding social castes: soldiers and housekeepers (Jarvis 1981, 1991; Lacey and Sherman 1991). Naked mole-rats are also extremely vocal rodents, both in terms of how often they vocalize and the number of different call types that

Thomas J. Park

tpark@uic.edu

1 Department of Life Sciences, The University of Tokyo, Tokyo, Japan

2 Department of Cognitive and Information Sciences, Chiba University, Chiba, Japan

3 Department of Biological Sciences, University of Illinois, Chicago, IL, USA

4 Department of Psychology, University of Maryland, College Park, MD, USA they produce. Within captive colonies, there is a continuous chatter of vocalizations, from which Pepper et al. (1991) identified 17 different call types associated with a variety of behavioral contexts. The naked mole-rats' high rate of vocalizing and their extensive vocal repertoire suggest that these animals rely heavily on auditory-vocal communication-a trait that is usually associated with good hearing capacity. However, a behavioral assay of their hearing (Heffner and Heffner 1993) revealed that, like other fossorial mammals, naked mole-rats have markedly higher auditory thresholds compared to non-fossorial, low frequency-hearing mammals (Brückmann and Burda 1997). Figure 1 shows the behavioral audiogram for naked mole-rats, and for comparison, an audiogram from gerbils (low frequency specialists). Note that thresholds for the naked mole-rats are substantially higher than those of the gerbils. Hence, there is an apparent incongruity for vocal communication in naked mole-rats; vocal signaling appears to be important to these animals, yet they also appear to have relatively poor hearing sensitivity.

This apparent incongruity is particularly bothersome in light of the overwhelming evidence that exists for the 


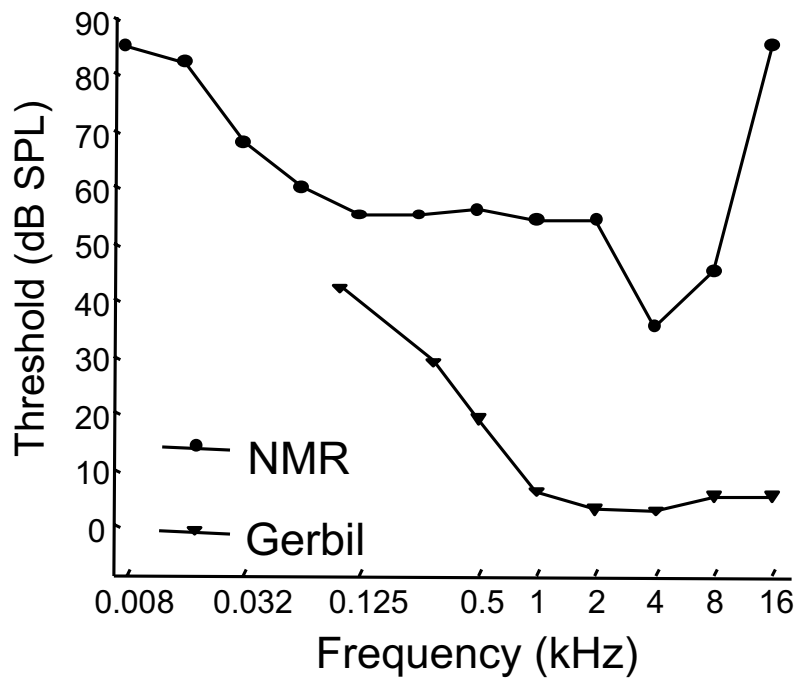

Fig. 1 Audiograms for naked mole-rats and gerbils. Behavioral audiogram for the naked mole-rat (closed circle) re-drawn from Heffner and Heffner (1993; data points for lowest two frequencies tested not shown). A behavioral audiogram for the gerbil (Ryan 1976; open triangle) is shown for comparison. $N M R$ naked mole-rat

co-evolution of coupled auditory and vocal systems across animal taxa (e.g., frog, cricket, fish, birds, bats; Ryan 1986; Gentner and Margoliash 2003; Sisneros and Bass 2003; Woolley and Moore 2011). Considering this potent evolutionary theme, one possible explanation pertaining to the naked mole-rat is that high auditory thresholds do not impair reception of species-specific vocal signals within the naked mole-rats' tunnel umwelt where low frequency sounds should propagate well, and environmental background noise should be minimal. Hence, theoretically, the naked mole-rat auditory system may be capable of receiving species-specific vocal signals at suprathreshold intensities over considerable distances.

We already know that hearing and vocal production are coupled within the spectral domain, both being in the low frequency range (Pepper et al. 1991; Heffner and Heffner 1993). What remains to be determined for the naked mole-rats is whether or not calls are produced with enough energy to propagate an appreciable distance through the tunnels at suprathreshold intensities. If not, vocal communication for this species may be limited to only relatively short distances. To address this issue, we first derived a physiological measure of hearing capacity to confirm the previous behavioral measures. We then characterized the acoustic properties of two naked mole-rat calls types (alarm calls and signature calls), focusing on both spectral content and on the intensity at which calls are produced. Finally, we measured the propagation properties of these calls traveling through tubes with the diameter of naked mole-rat tunnels.

\section{Materials and methods}

The original research reported herein was performed under guidelines established by the Institutional Animal Care and Use Committees at the University of Illinois at Chicago, the University of Maryland, Chiba University, and the RIKEN Brain Science Institute.

\section{Auditory brainstem response}

We measured auditory brainstem responses from four naked mole-rats (NMR, Heterocephalus glaber) and two mongolian gerbils (Meriones unguiculatus). The naked mole-rats included one breeding female, one breeding male, and two non-breeding adult females. The ages of these animals were 6.3 years, 3.4 years, 1 year, and 6 years, respectively. Captive naked mole-rats can live to be over 30 years old, and we consider 1-6 year olds to be young adults (Buffenstein et al. 2012). The gerbils were between 2 and 8 months, also considered to be young adults (mongolian gerbils can live to be 5 years old).

All animals were sedated with subcutaneous injection of Ketamine $(35-50 \mathrm{mg} / \mathrm{kg}$ ) and Xylazine $(8 \mathrm{mg} / \mathrm{kg}$ ) prior to electrode placement. Animals remained relatively motionless for up to $75 \mathrm{~min}$. Body temperature was maintained at $30 \pm 0.5{ }^{\circ} \mathrm{C}(\mathrm{NMR})$ and $37 \pm 0.5{ }^{\circ} \mathrm{C}$ (gerbils) with a heating pad and monitored with a thermistor probe (Frederick Haer and Co., Model 40-90). Note that naked mole-rats are poikilotherms, and in nature, their body temperature would reflect the ambient temperature in their tunnels which is usually about $30{ }^{\circ} \mathrm{C}$ (Bennett and Faulkes 2000).

Standard platinum alloy, subdermal needle electrodes, (Grass F-E2; West Warwick, RI, USA) were placed just under the skin high at the vertex (active), directly behind the right ear canal (the ear ipsilateral to the speaker, reference) and behind the canal of the ear contralateral to stimulation (ground). Shielded electrode leads were twisted together to reduce electrical noise through common mode rejection.

The stimulus presentation, ABR acquisition, equipment control, and data management were coordinated using a Tucker-Davis Technologies (TDT, Gainesville, FL, USA) modular rack-mount system controlled by an optical cable-linked 350-MHz Pentium PC containing a TDT AP2 Digital Signal Process board and running TDT 'BIOSIG' software. Sound stimuli were generated using TDT 'SIGGEN' software and fed through a DA1 digitalanalog converter, a PA4 programmable attenuator, and a HB6 transducer which directly drove the JBL Professional Series speaker (Model 2105H, James B Lansing Sounds Inc.). The electrodes were connected to the TDT HS4 
Headstage that amplifies and digitizes the signal before sending it over fiber optic cables to the TDT DB4 Digital Biological Amplifier. This amplifier allows additional filtering and gain to be added. A TDT TG6 timing generator synchronized the $\mathrm{A} / \mathrm{D}$ and $\mathrm{D} / \mathrm{A}$ conversion.

Stimulus intensities were measured in the free field by placing the $1 / 2$-in. microphone of a sound level meter (System 824; Larson Davis, Inc. Provo, UT, USA) at the approximate position of the animal's ear ( $30 \mathrm{~cm}$ from speaker). Tones were played continuously using the TDT BIOSIG program and measured using the fast A-weighted scale on the SLM. For values below $1 \mathrm{kHz}$, the A-filter values were corrected for the filter. To determine the intensity of the short duration click, we used the peak equivalent SPL of the click. This was determined using an oscilloscope and noting the peak-to-peak voltage of the click. A test tone, e.g., a $1 \mathrm{kHz}$ tone, was played and adjusted until the peak-to-peak voltage was the same as it was for the click. The SPL required to match the amplitude of the click, as indicated at the sound level meter, was the peak equivalent SPL (dB pSPL) of the click stimulus.

\section{Stimuli}

Subjects were presented with multiple intensity stimulus trains that varied in frequency and intensity (see BrittanPowell et al. 2002, 2005; Brittan-Powell and Dooling 2004; Wright et al. 2004). Each train consisted of 9 tone bursts or clicks. Stimulus trains were presented at a rate of $4 / \mathrm{s}$ and progressively increased in intensity. The click trains consisted of rectangular-pulse broadband clicks were $0.1 \mathrm{~ms}$ $(100 \mu \mathrm{s})$ in duration with $25 \mathrm{~ms}$ inter stimulus interval (ISI). Each individual tone burst was $5 \mathrm{~ms}$ in duration $(1 \mathrm{~ms}$ rise/ fall COS2) with a $20 \mathrm{~ms}$ ISI. Short rise times were used because they have less of an effect on ABR latency and wave morphology (Hecox et al. 1976; Kodera et al. 1977, 1979), and nonlinear gating methods, such as COS2, provide narrowband amplitude spectrum and considerable reduction of amplitudes of side lobes that can detract from frequency specificity (Robier et al. 1992). The tone bursts used were $0.25,0.5,1,2,4,8$ and $10 \mathrm{kHz}$, with intensities spanning a 40-dB range in ascending order of $5 \mathrm{~dB}$ steps (e.g., started at $70 \mathrm{~dB}$ and increasing to $110 \mathrm{~dB}$ or starting at $30 \mathrm{~dB}$ and increasing to $70 \mathrm{~dB}$ ). Tone burst spectra were generated using 1024-point fast Fourier transform (FFT) and showed all harmonics were at least $20 \mathrm{~dB}$ down from the peak of the frequency of interest.

Each ABR represents the average response of 600 stimulus presentations (300 averages for each polarity/phase were added together to cancel the cochlear microphonic), sampled at $20 \mathrm{kHz}$ for $235 \mathrm{~ms}$ following onset of the stimulus. The biological signal was amplified $(\times 100 \mathrm{~K})$ and notch filtered at $60 \mathrm{~Hz}$ with the DB4 Digital Biological Amplifier during collection; the averaged signal was bandpass filtered below $30 \mathrm{~Hz}$ and above $3000 \mathrm{~Hz}$ after collection using BIOSIG.

Thresholds were estimated using the visual detection method (Brittan-Powell and Dooling 2004; Brittan-Powell et al. 2005): the lowest intensity at which a response could be detected visually on the trace, regardless of wave, or $2.5 \mathrm{~dB}$ below the lowest intensity that elicited a measurable response (examples will be given below in Fig. 2 of the "Results").

\section{Analysis of vocalizations}

Recordings were made in a wooden box $(45 \times 30 \times 30 \mathrm{~cm})$ lined with acoustic foam panels (thickness: $2 \mathrm{~cm}$ ) in a sound attenuating chamber. We placed a condenser microphone (SONY ECM-MS957) $20 \mathrm{~cm}$ above the floor of the recording box. The microphone was connected to a Windows compatible PC through a preamplifier and a sound card (ONKYO SE-U77). Recordings to the hard disk were carried out via Avisoft-SASlab Pro (Avisoft Bioacoustics, Germany) set at a $44.1-\mathrm{kHz}$ sampling rate and a 16-bit resolution, and the recorded sound was stored as a wave file. The animals we recorded from were all 9-15 months old and similarly sized, and we presumed that they were all in the same caste (i.e. "workers" not "soldiers"). Signature calls were recorded by gently pushing the animal's back (Yosida et al. 2007; Yosida and Okanoya 2009). Alarm calls were recorded by grabbing the animal's tail. At least five instances of each call were recorded per animal. Spectrograms of all calls were produced with a $240 \mathrm{~Hz}$ analysis bandwidth using Avisoft.

\section{Sound pressure measurements}

While recording these vocalizations, the microphone of the sound level meter (RION) was placed $20 \mathrm{~cm}$ above the floor of the recording box and real-time measurement of the sound intensity of each recorded call was performed. The sound level meter was set at an A-weighted scale and fast integration time. Since the animal moved about the floor of the recording chamber, exact distance between the animal and the tip of the microphone could not be determined. We estimated the average distance to be $24 \mathrm{~cm}$. Based on this, the sound pressure level at $12 \mathrm{~cm}$ from the animal's mouth was estimated to be $6 \mathrm{~dB}$ higher than that actually recorded.

\section{Tunnel diameter and transmission characteristics}

To assess how sound propagation through a naked molerat tunnel would affect the characteristics of calls, we first had to derive an estimate of naked mole-rat tunnel diameter. We accomplished this by allowing naked mole-rats to dig tunnels within a 1 cubic meter tub of compacted, sterilized soil for $48 \mathrm{~h}$. For this procedure, we used a small colony of 
Fig. 2 ABR waveforms evoked by a $2 \mathrm{kHz}$ tone train presented at different intensities for a gerbil and a naked mole-rat. Arrows indicate thresholds for this frequency, $22.5 \mathrm{~dB}$ for the gerbil and $52.5 \mathrm{~dB}$ for the naked mole-rat. Due to the differences in thresholds, the range of intensities shown are different for the two animals. Note that the scale bars ( $\mathrm{uV})$ also differ between species because ABR waveform amplitudes for the naked molerat were smaller in general compared to the gerbil. This species difference was consistent across all animals tested
Gerbil
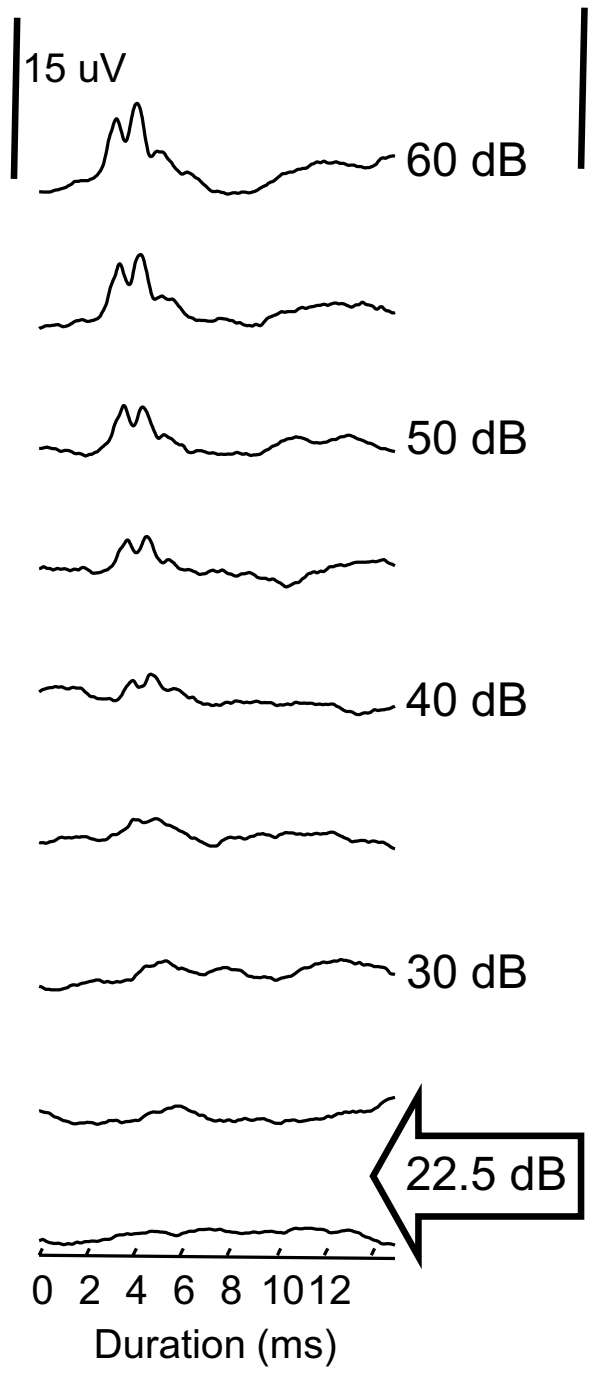

Naked mole-rat

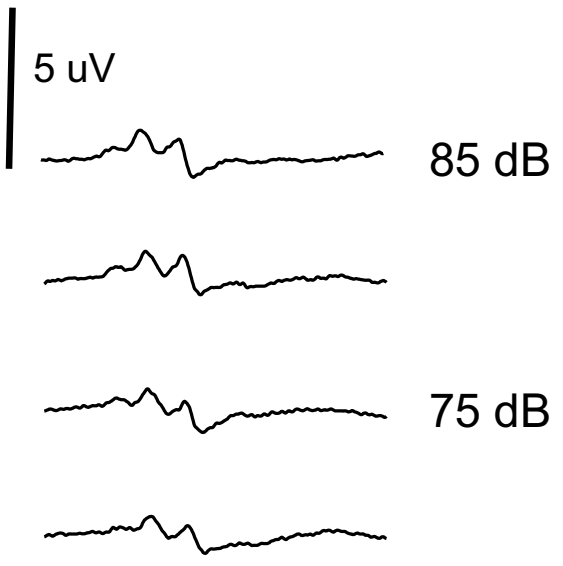

$65 \mathrm{~dB}$
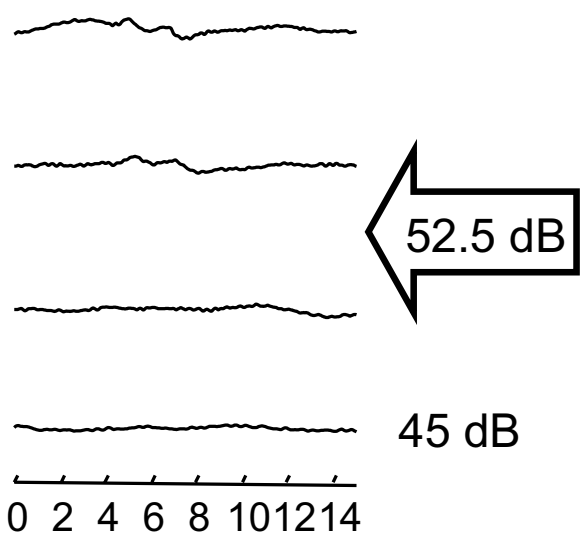

$45 \mathrm{~dB}$

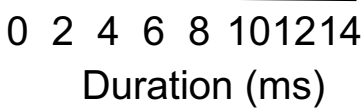

rice paper. Tones were generated with the same equipment as those for testing ABRs. For assessed transmission characteristics, we used tones with a duration of $200 \mathrm{~ms}$ with $10 \mathrm{~ms}$ rise/fall times. Two of the frequencies we chose were similar to the fundamental frequencies of alarm calls $(0.5 \mathrm{kHz})$ and signature calls $(3.1 \mathrm{kHz}) .10 .6 \mathrm{kHz}$ was chosen as a third frequency so that we would have data for a frequency at the upper limit of the naked mole-rat's hearing ability (Heffner and Heffner 1993). Tones and calls were played with a peak amplitude of $80 \mathrm{~dB}$. The plain PVC pipe is relatively non-sound absorbing so we also measured transmission characteristics through pipe lined with soft rice paper to simulate a relatively greater absorbing environment. Note that in burrows, audible sounds appear to propagate much more through the air in the burrow than through the soil (Narins et al. 1997). 


\section{Results}

Here, we report on the hearing capacity of naked molerats, the characteristics of their alarm and signature calls, and the propagation characteristics of these calls through tubes with a diameter based on naked mole-rat tunnels.

\section{Auditory brainstem response (ABR)}

We measured the ABR from four naked mole-rats and two gerbils. Gerbils were chosen as a comparison species because there is an abundance of both ABR and behaviorally derived audiogram data in the literature for this species (e.g., McFadden et al. 1996).

ABR waveforms were similar for naked mole-rats and gerbils except that the amplitude of the naked mole-rat response was only about $25 \%$ that of the gerbil. Figure 2 shows ABR waveforms evoked by different intensities from a naked mole-rat and a gerbil tested with $2 \mathrm{kHz}$ tones. Visual examination of the waveforms showed 2-3 prominent peaks that occurred within the first $8 \mathrm{~ms}$ after sound reached the animal's external ear canal. As with all animals tested to date (e.g., Hecox and Galambos 1974; Starr and Achor 1975; Picton et al. 1976), increasing the intensity of stimulation, caused two major changes: latencies to all waves decreased, and amplitudes of all waves increased.

As mentioned in the "Materials and methods" section above, thresholds for a given frequency were estimated visually from the waveforms. For the data in Fig. 2, we estimated a threshold for the gerbil to be $22.5 \mathrm{~dB}$, and we estimated threshold for the naked mole-rat to be $52.5 \mathrm{~dB}$ (both indicated by arrows).

We used ABR data from across frequencies and intensities to construct audiogram curves, which are shown in Fig. 3. Each point on an audiogram curve corresponds to a threshold response for a particular frequency. For example, the $0.25 \mathrm{kHz}$ tone evoked a threshold response from the naked mole-rats at an average intensity of $75 \mathrm{~dB}$, and from the gerbils at an average intensity of $40 \mathrm{~dB}$.

The audiograms from both species had a characteristic U-shaped function, but the naked mole-rats had consistently higher thresholds compared to the gerbils. On average, thresholds for the naked mole-rats were between 25 and $50 \mathrm{~dB}$ higher than those of the gerbils, which is consistent with the differences observed in the behavioral audiograms shown in Fig. 1.

Comparison to the behaviorally derived audiograms presented in Fig. 1 shows that the ABR derived curves are elevated by about $15-25 \mathrm{~dB}$ for both naked mole-rats and gerbils. This overall difference between behaviorally and

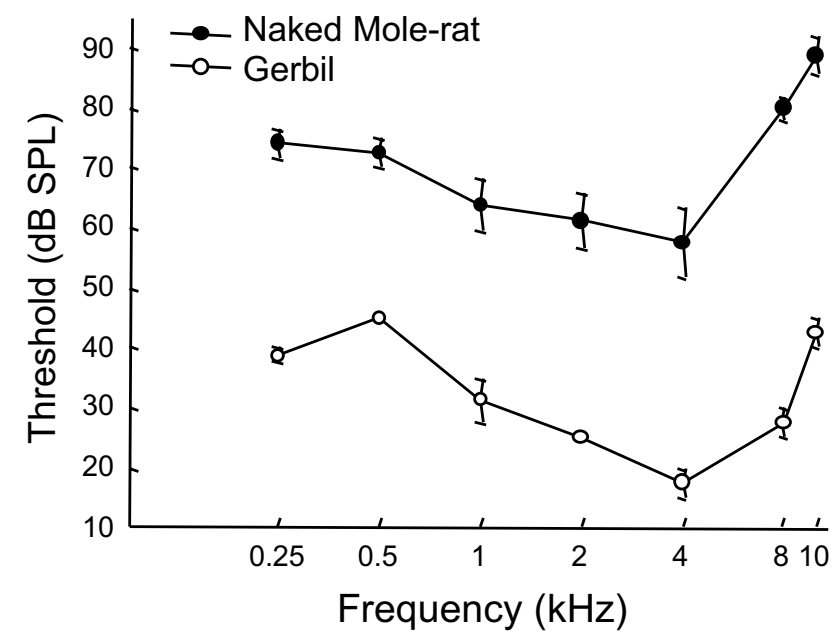

Fig. 3 ABR-derived audiograms for four naked mole-rats and two gerbils. Error bars are s.e.m.

ABR derived audiograms is consistent with previous data comparing the two techniques (e.g., Gorga et al. 1988; Werner et al. 1993; Brittan-Powell et al. 2002). Since the $\mathrm{ABR}$ is an onset response, much of the difference may be attributed to differences in temporal integration (Brittan-Powell et al. 2002). Given the consistent difference between behaviorally and ABR derived audiograms across a wide range of species, we interpret our present naked mole-rat ABR audiogram to be in good agreement with the previously reported behavioral audiogram.

\section{Analysis of naked mole-rat vocalizations}

We recorded five alarm calls and five signature calls from each of five individual naked mole-rats. We focused on these two call types because they are acoustically distinct and because they are associated with very different behavioral contexts (Pepper et al. 1991). The alarm call (also referred to as the "grunt" call) functions as a colony defense call (Pepper et al. 1991). The signature call (also referred to as the "soft chirp" call) is the most common vocalization, and appears to function as a short distance, close contact call, usually emitted when animals touch one another (Pepper et al. 1991; Yosida et al. 2007). Within individuals these calls are emitted with a high degree of reproducibility. Across individuals they vary widely in duration but remain very similar in spectral content and intensity (Yosida and Okanoya 2009).

Spectrograms of alarm and signature calls from different individuals are displayed in Fig. 4. For the alarm calls (Fig. 4a), the fundamental frequency was about $300 \mathrm{~Hz}$. Each call had a fundamental plus 4-5 harmonics, with call length ranging from 100 to $150 \mathrm{~ms}$. Alarm calls were produced at an average intensity of $85.9 \mathrm{~dB}$ (grand average 
Fig. 4 shows representative spectrograms and average power spectra from alarm calls and signature calls. a Alarm calls from five different individual naked mole-rats, five calls each (each row is from one animal). The scale bar in the upper left is $100 \mathrm{~ms}$. b Signature calls from five different individual naked mole-rats, five calls each (each row is from one animal). The scale bar in the upper left is $100 \mathrm{~ms}$. c Average power spectra from five animals, five calls each for alarm and signature calls a Alarm calls

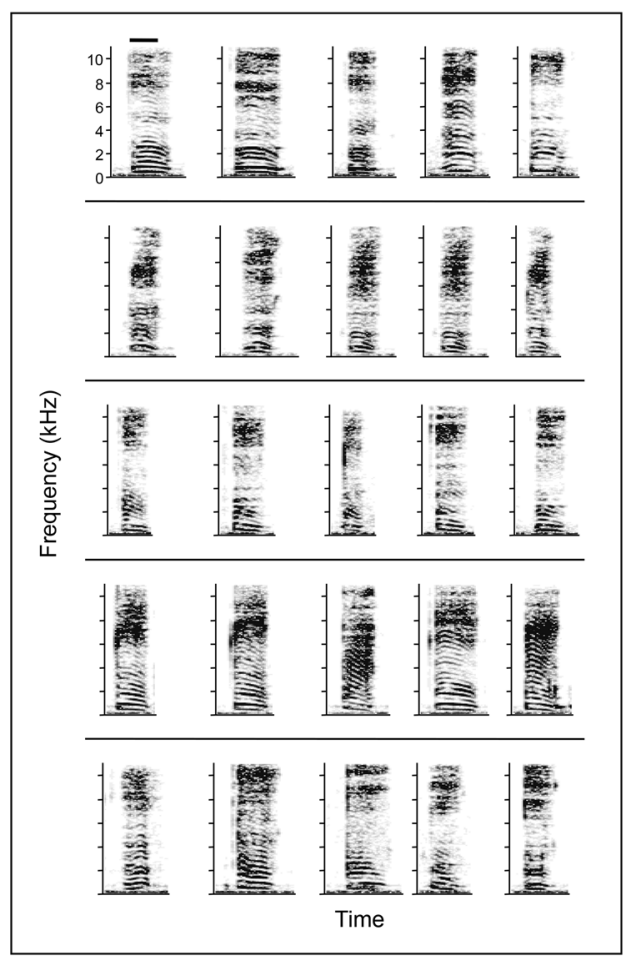

b Signature calls

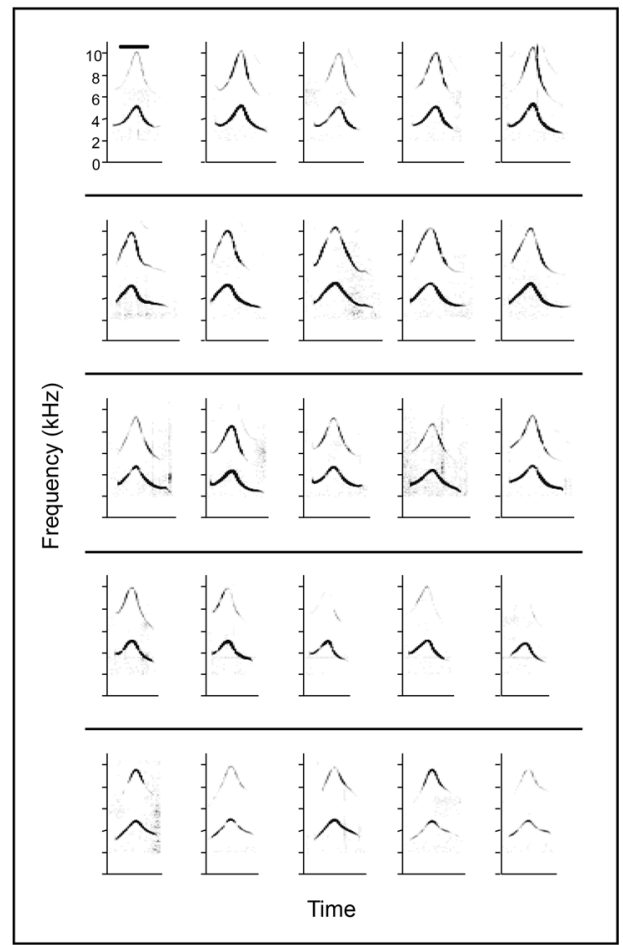

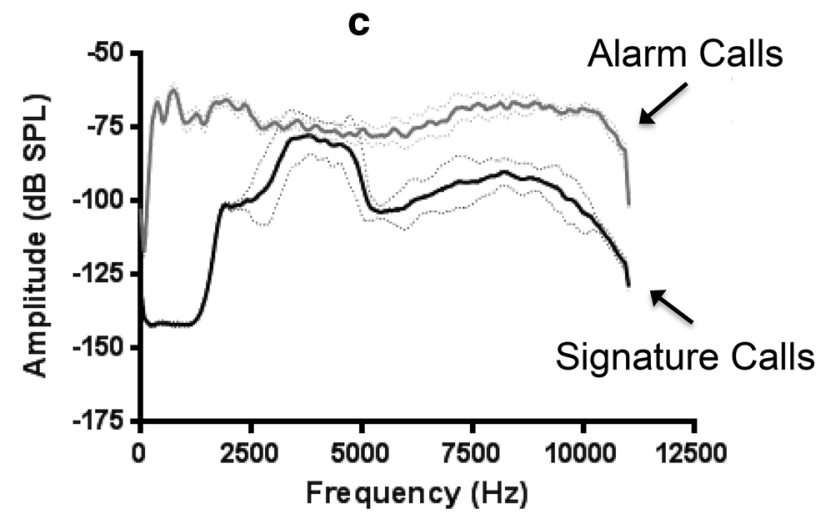

of five animals, five calls each, $\mathrm{SD}=0.93$ ), measured at $20 \mathrm{~cm}$ from the animal. For the signature calls (Fig. 4b), the fundamental frequency was between 3 and $5 \mathrm{kHz}$ with a characteristics frequency modulation that first went up and then down. Each call had a fundamental plus 1-3 harmonics, with call length ranging from 100 to $200 \mathrm{~ms}$. The signature calls were produced at an average intensity of $63.4 \mathrm{~dB}$ (grand average of five calls from five animals, $\mathrm{SD}=0.60)$ measured $20 \mathrm{~cm}$ from the animal. To summarize the characteristics of the alarm and signature calls across individuals, we calculated the average power spectrum from five animals, five calls each which is presented in Fig. 4c.

\section{Transmission characteristics of simulated tunnels}

Naked mole-rats in our laboratory dug tunnels with an average diameter of $4.03 \mathrm{~cm} \mathrm{(35} \mathrm{measurements} \mathrm{over} 5 \mathrm{~m}$ of tunnel, $\mathrm{SD}=0.884 \mathrm{~cm}$ ), which corresponds closely to measurements made from excavated tunnels in the field (Jarvis and Bennett 1991). Attenuation level of a sound in a tunnel would depend on at least two factors. First, since the tunnel limits the diffusion of sound energy proportional to the square of the distance from the source, an ideal tunnel should reduce the amount of attenuation that follows the inverse square law. Second, since the surface of the tunnel would reflect and/or absorb the sound energy, the 
physical property of the sound would give excess attenuation. Because of the complexity of this acoustics, we can not theoretically predict attenuation levels of the calls broadcast in the tunnel of naked mole-rats.

To measure actual attenuation of the sound in a simulated naked mole-rat tunnel, we played tones and calls through a $4 \mathrm{~cm}$ PVC pipe using a variety of pipe lengths, and two inner surface textures: plain PVC pipe and pipe lined with soft, sound absorbent rice paper. While the pipe is obviously an idealized tube compared to actual mole-rat tunnels, both retain the principles of a tube structure. The curves in Fig. 5 show how the pipe affected the intensity of tones as a function of distance and tone frequency for three representative frequencies. The set of dashed curves were measured with the plain pipe, and the set of solid curves were measured with the pipe lined with soft rice paper. In both cases, higher frequencies showed greater attenuation over distance than lower frequencies, as was expected from the low-pass filter properties of tubes.

Tones of $0.5 \mathrm{kHz}$, roughly the frequency of peak energy for the alarm calls, were attenuated by about $10 \mathrm{~dB}$ over $5 \mathrm{~m}$ through both the plain PVC pipe (dashed curves), and through pipe lined with soft rice paper (solid curves). Knowing that the naked mole-rats have a behavioral hearing threshold of $55 \mathrm{~dB}$ for $0.5 \mathrm{kHz}$ (Fig. 1), and that they produce alarm calls with an average intensity of $86 \mathrm{~dB}$ at $20 \mathrm{~cm}$, we estimate that an alarm call should be able to travel through the tunnel for about $15 \mathrm{~m}$ before call intensity drops below threshold.

A higher frequency of $3.1 \mathrm{kHz}$, roughly the frequency of peak energy for the signature calls, was attenuated by 8 $\mathrm{dB}$ (plain PVC pipe, open symbols) to $24 \mathrm{~dB}$ (pipe lined

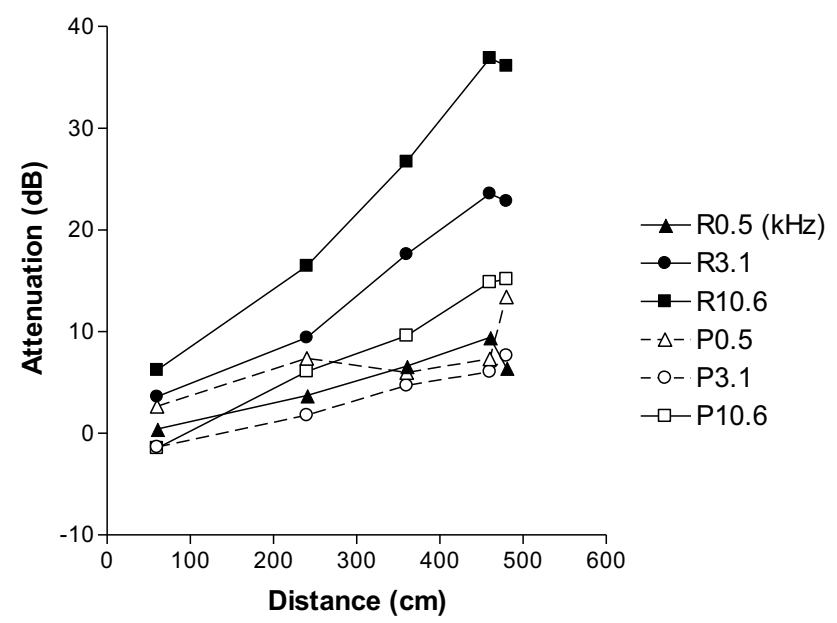

Fig. 5 Attenuation through pipes as a function of distance and frequency. Tones with frequencies of $0.5,3.1$, and $10.6 \mathrm{kHz}$ were played through plain PVC pipe (dashed lines with open symbols, "P" in the legend) or played through PVC pipe lined with soft rice paper (solid lines with solid symbols, " $R$ " in the legend) with soft rice paper, solid symbols) over $5 \mathrm{~m}$. Hence, we estimate that signature calls, which are produced at $63 \mathrm{~dB}$, should travel through the tunnel between 3 and $8 \mathrm{~m}$ before call intensity drops below the threshold of $50 \mathrm{~dB}$. Higher frequency tones were attenuated more.

To determine how passing through the tubes would affect the spectral characteristics of calls, we played both call types through the pipes. Figure 6 shows the average power spectra of one representative alarm call and signature call after transmission through $3.2 \mathrm{~m}$ of pipe. Although the sound morphology was maintained for the alarm call, it was more degraded for the signature call.

\section{Discussion}

Our main conclusion is that high intensity, low frequency alarm calls are audible over long distances through tunnels while lower intensity, higher frequency signature calls are audible over much shorter distances. This result is in agreement with basic acoustics. In both cases (alarm and signature calls), the distances that these calls propagate are well matched to their function. Also, even though the alarm and signature calls were substantially different from one another in spectral characteristics, both call types are well within the range of best hearing for the naked mole-rats.

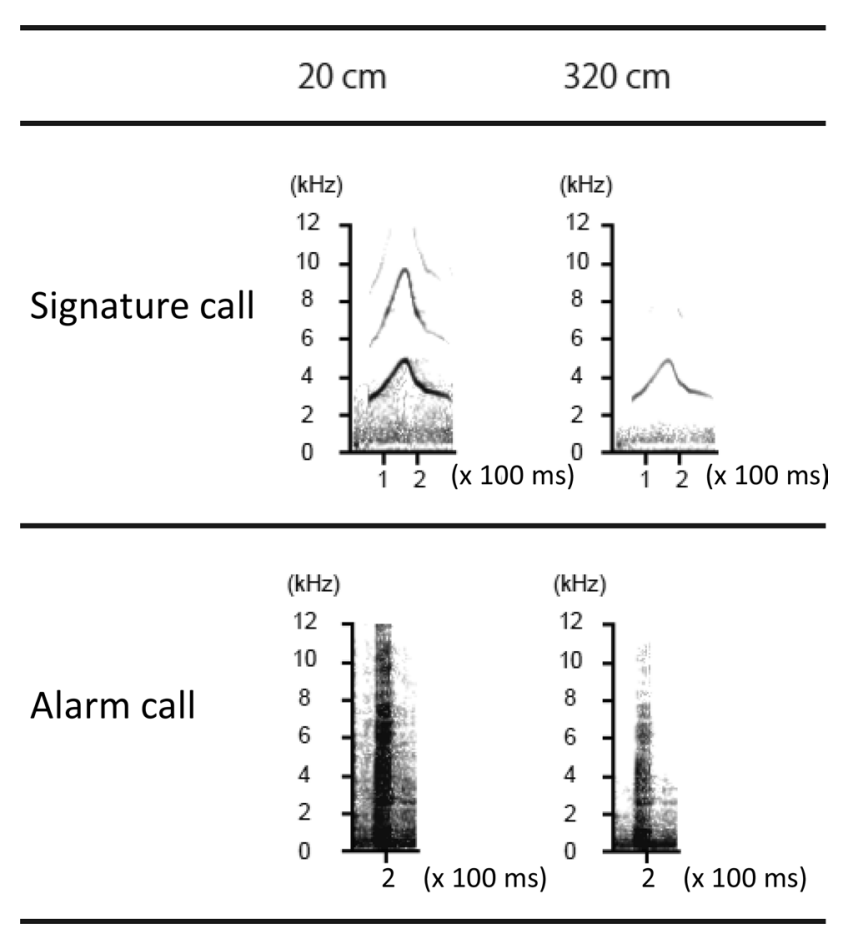

Fig. 6 Spectrograms of a signature calls, and an alarm call after transmission through 0.2 and $3.2 \mathrm{~m}$ pipes 


\section{Propagation through tunnels}

There are obvious drawbacks to measuring sound characteristics through PVC pipe instead of actual burrows in the field. Thus, while our conclusion that alarm calls propagate substantially farther than signature calls is well supported, our quantitative measures of attenuation through PVC pipes is likely an underestimate of what happens in actual burrows. However, data published by Lange et al. (2007) on sound propagation through actual burrows of Fukomys mole-rats is not that different from what we observed with the PVC pipe, particularly for low frequency sounds. For $0.5 \mathrm{kH}$, we found attenuation through the $4 \mathrm{~cm}$ pipe to be $2 \mathrm{~dB} / \mathrm{m}$. Lange et al. tested a similar frequency $(0.4 \mathrm{kH})$ through burrows with an average diameter of $5 \mathrm{~cm}$ and found attenuation to be $3.1 \mathrm{~dB} / \mathrm{m}$. For higher frequencies (e.g. $3.1-3.2 \mathrm{kH}$ ), Lange et al. found considerably more attenuation through the $5 \mathrm{~cm}$ burrow $(50.4 \mathrm{~dB} / \mathrm{m})$ compared to what we found with the PVC pipe $(5 \mathrm{~dB} / \mathrm{m})$. We recognize that the difference between the findings of Lange et al. and the present report for a higher frequency are large but we do not have an explanation at the time to account for it. It is notable that attenuation measured by Lange et al. through the $5 \mathrm{~cm}$ burrow was the greatest they reported among eight burrows of various diameters and species. It is also notable that Lange et al. showed that curves in actual tunnels probably increase attenuation of sounds. Brett (1991) reported that naked mole-rat burrows have curves and branches. Another factor that would potentially increase attenuation in actual burrows would be the effect of colony mates blocking the burrows with their bodies. We also point out that while environmental background noise is low in burrows, the animals themselves could make substantial "noise" from vocalizing, digging, and moving about the burrows, which could potentially interfere with communication.

\section{Auditory thresholds}

The ABR-derived measure of auditory sensitivity confirmed that the naked mole-rats have best hearing for low frequencies with high thresholds overall. These results are consistent with a previous, behaviorally derived audiogram for this species (Heffner and Heffner 1993). We also found that the amplitude of the ABR waveform was substantially smaller for the naked mole-rats compared to gerbils (and other mammals). We do not yet know why, or if, this is a common feature across subterranean mammals or particular to naked mole-rats.

Low frequency hearing appears to be a common characteristic of subterranean hearing, and low frequency vocalizations are common for these species as well (Bruns et al. 1988; Burda et al. 1992; Credner et al. 1997; Nevo 1999). This pattern has been reported for the Zambian mole-rat
(Africa; Brückmann and Burda 1997; Credner et al. 1997), the blind mole-rat (Europe; Nevo et al. 1987; Heth et al. 1988; Bronchti et al. 1989), and the coruro (South America; Veitl et al. 2000; Begall et al. 2004).

A close relationship between tunnel propagation characteristics, hearing sensitivity, and call spectra may be common to other subterranean mammals. The blind molerat (Spalax ehrenbergi), a solitary-living species native to the Middle East, emits low frequency calls in the range of $0.5 \mathrm{kHz}$ (Heth et al. 1986; Nevo et al. 1987). The range of most sensitive hearing for this species is $0.5-1.5 \mathrm{kHz}$ (Bruns et al. 1988; Bronchti et al. 1989), and their tunnels show best propagation for frequencies around $0.5 \mathrm{kHz}$ (Heth et al. 1986).

Despite the good match between best hearing sensitivity and call spectrum, the naked mole-rats' overall high thresholds would greatly restrict the distance over which auditory-vocal communication would be effective in an open environment. However, naked mole-rats are never in an open environment. Rather, they live their entire lives within a tube-like burrow system that facilitates call propagation, much like a speaking tube on navy ships and playgrounds (Elliot and Foulkes 2011). There are a number of factors that affect sound propagation through tube-like structures, including temperature, humidity, and surface texture, but the most important factors are the diameter of the tube, and the frequency of the sound (Lange et al. 2007). Our measurements indicate that the low frequency alarm calls of naked mole-rats propagate well through tubes with a diameter based on naked mole-rat tunnels. Hence, the low background noise and the propagation characteristics of their tunnels, together with calls produced at high intensities, could compensate to a large extent for the naked mole-rats' comparatively high auditory thresholds.

In contrast to alarm calls, signature calls, which are produced at higher frequencies and lower intensities, propagate over much shorter distances before becoming degraded and inaudible. However, this appears to be consistent with the behavioral context in which signature calls are used. Naked mole-rats consistently emit signature calls when touched by another mole-rat, suggesting a function in close contact communication.

\section{A coupled auditory-vocal communication system}

A hallmark feature of acoustic communication systems across taxa is co-evolution of coupled signal production and auditory reception characteristics. Within their tunnels, the same phenomenon appears to apply to subterranean mammals. The interesting thing about these species is that the coupling takes place with higher auditory thresholds than are typical for the range of best hearing sensitivity of other mammals. Burda et al. (1992) have 
argued that the limiting factors for hearing sensitivity in subterranean mammals result from constraints on head size, and that given these constrains, middle ear structures are actually well adapted for low frequency, subterranean hearing. Furthermore, Lange et al. (2007) have suggested that decreased sensitivity in subterranean mammals may be a protective adaptation. Those authors measured attenuation of sound in burrows of Fukomys mole-rats in the field and they found that low frequencies not only propagated well, they could be substantially amplified.

Alternatively, in a closed tunnel system there may be less pressure to maintain the same high degree of auditory sensitivity typical of non-subterranean mammals. Because the naked mole-rats generate their calls at such relatively high intensities, it is interesting to speculate that the cost of maintaining high auditory sensitivity may outweigh the energetic cost of producing relatively high-intensity vocalizations. In this regard, another interesting feature of subterranean life that is related to high-intensity vocalizations is that the threat of predation based on predators hearing the communication calls is greatly reduced for subterraneans compared to surface dwelling rodents.

Acknowledgements We thank Dr. Kohta Kobayashi, Miki Takahashi, Masayuki Ohtake, and Maki Ikebuchi for their effort in setting up the naked mole-rat colony at Chiba University. We thank Symmorron Bailey for excellent animal care of the naked mole-rat colony at the University of Illinois at Chicago. We thank two anonymous reviewers for thoughtful suggestions. This research was supported in part by NSF Grant 1655494 (TJP).

Open Access This article is distributed under the terms of the Creative Commons Attribution 4.0 International License (http://creativeco mmons.org/licenses/by/4.0/), which permits unrestricted use, distribution, and reproduction in any medium, provided you give appropriate credit to the original author(s) and the source, provide a link to the Creative Commons license, and indicate if changes were made.

\section{References}

Artwohl J, Hill T, Comer CM, Park TJ (2002) Naked mole-rats: unique opportunities and husbandry challenges. Lab Anim 31:32-36. https://doi.org/10.1038/5000156

Begall S, Burda H, Schneider B (2004) Hearing in coruros (Spalacopus cyanus): special audiogram features of a subterranean rodent. J Comp Physiol A Neuroethol Sens Neural Behav Physiol 190:963-969. https://doi.org/10.1007/s00359-004-0552-1

Bennett NC, Faulkes CG (2000) African mole-rats: ecology and eusociality. Cambridge University Press, Cambridge

Brett RA (1991) The ecology of naked mole-rat colonies: burrowing, food, and limiting factors. In: Sherman PW, Jarvis JUM, Alexander RD (eds) The biology of the naked mole-rat. Princeton University Press, Oxford, pp 97-136

Brittan-Powell EF, Dooling RJ (2004) Development of auditory sensitivity in budgerigars (Melopsittacus undulatus). J Acoust Soc Am 115:3092-3102. https://doi.org/10.1121/1.1739479
Brittan-Powell EF, Dooling RJ, Gleich O (2002) Auditory brainstem responses in adult budgerigars (Melopsittacus undulatus). J Acoust Soc Am 112:999-1008. https://doi.org/10.1121/1.1494807

Brittan-Powell EF, Lohr B, Hahn DC, Dooling RJ (2005) Auditory brainstem responses in the eastern screech owl: an estimate of auditory thresholds. J Acoust Soc Am 118:314-321. https://doi. org/10.1121/1.1928767

Bronchti G, Heil P, Scheich H, Wollberg Z (1989) Auditory pathway and auditory activation of primary visual targets in the blind molerat (Spalax ehrenbergi): I. 2-Deoxyglucose study of subcortical centers. J Comp Neurol 284:253-274. https://doi.org/10.1002/ cne. 902840209

Brückmann G, Burda H (1997) Hearing in blind subterranean Zambian mole-rats (Cryptomys sp.): collective behavioural audiogram in a highly social rodent. J Comp Physiol A 181:83-88. https://doi. org/10.1007/s003590050

Bruns V, Muller M, Hofer W, Heth G, Nevo E (1988) Inner ear structure and electrophysiological and audiograms of the subterranean mole-rat Spalax ehrenbergi. Hear Res 33:1-10. https://doi. org/10.1016/0378-5955(88)90017-2

Buffenstein R, Park T, Hanes M, Artwohl JE (2012) Naked mole rat. In: Suckow MA, Stevens KA, Wilson RP (eds) The laboratory rabbit, guinea pig, hamster, and other rodents. Elsevier, London, pp 1055-1074

Burda H, Bruns V, Hickman CG (1992) The ear in subterranean insectivora and rodentia in comparison with ground-dwelling representatives. J Morphol 214:49-61. https://doi.org/10.1002/ jmor.1052140104

Credner S, Burda H, Ludescher F (1997) Acoustic communication underground: vocalization characteristics in subterranean social mole-rats (Cryptomys sp., Bathyergidae). J Comp Physiol 180:245-255. https://doi.org/10.1007/s003590050

Elliot WJ, Foulkes JT (2011) A survey of residential "speaking tubes". J Acoust Soc Am 130:2318. https://doi.org/10.1121/1.3654266

Gentner T, Margoliash D (2003) The neuroethology of vocal communication: perception and cognition. In: Simmons AM, Popper AN, Fay RR (eds) Acoustic communication. Springer, New York, pp 324-386

Gorga M, Kaminski J, Beauchaine K, Jesteadt W (1988) Auditory brainstem responses to tone bursts in normally hearing subjects. J Speech Hear Res 31:89-97. https://doi.org/10.1044/jshr.3101.87

Hecox K, Galambos R (1974) Brain stem auditory evoked responses in human infants and adults. Arch Otolaryngol 99(1):30-33. https:// doi.org/10.1001/archotol.1974.00780030034006

Hecox K, Squires N, Galambos R (1976) Brain stem evoked responses in man: I. Effects of stimulus rise/fall time and duration. $\mathrm{J}$ Acoust Soc Am 60:1187-1192. https://doi.org/10.1121/1.381194

Heffner RS, Heffner HE (1993) Degenerative hearing and sound localization in naked mole-rats (Heterocephalus glaber), with an overview of central auditory structures. J Comp Neurol 331:418-433. https://doi.org/10.1002/cne.903310311

Heth G, Frankenberg E, Nevo E (1986) Adaptive optimal sound for vocal communication in tunnels of a subterranean mammal (Spalax ehrenbergi). Experientia 42:1287-1289. https://doi. org/10.1007/BF01946426

Heth G, Frankenberg E, Nevo E (1988) "Courtship" call of subterranean mole rats (Spalax ehrenbergi): physical analysis. J Mammal 69:121-125. https://doi.org/10.2307/1381755

Jarvis JUM (1981) Eusociality in a mammal: cooperative breeding in naked mole-rat colonies. Science 212:571-573. https://doi. org/10.1126/science.7209555

Jarvis JUM (1991) Reproduction of naked mole-rats. In: Sherman PW, Jarvis JUM, Alexander RD (eds) The biology of the naked molerat. Princeton University Press, Princeton, pp 384-425

Jarvis JUM, Bennett NC (1991) Ecology and behavior of the family bathyergidae. In: Sherman PW, Jarvis JUM, Alexander RD (eds) 
The biology of the naked mole-rat. Princeton University Press, Princeton, pp 66-96

Kodera K, Yamane H, Yamada O, Suzuki JI (1977) The effects of onset, offset, and rise-decay times of tone bursts on brain stem responses. Scand Audiol 6:205-210. https://doi.org/10.3109/0105039770 9043122

Kodera K, Hink RF, Yamada O, Suzuki JI (1979) Effects of rise time on simultaneously recorded auditory-evoked potentials from the early, middle, and late ranges. Audiology 18:395-402. https://doi. org/10.3109/00206097909070065

Lacey EA, Sherman PW (1991) Social organization of naked mole-rat colonies: evidence for divisions of labor. In: Sherman PW, Jarvis JUM, Alexander RD (eds) The biology of the naked mole-rat. Princeton University Press, Princeton, pp 275-336

Lange S, Burda H, Wegner RE, Dammann P, Begall S, Kawalika M (2007) Living in a "stethoscope": burrow-acoustics promote auditory specializations in subterranean rodents. Naturwissenschaften 94(2):134-138. https://doi.org/10.1007/s00114-006-0168-0

McFadden SL, Walsh EJ, McGee J (1996) Onset and development of auditory brainstem responses in the Mongolian gerbil (Meriones unguiculatus). Hear Res 100:68-79. https://doi.org/10.1016/03785955(96)00108-6

Narins PM, Lewis ER, Jarvis JJ, O'Riain J (1997) The use of seismic signals by fossorial southern African mammals: a neuroethological gold mine. Brain Res Bull 44:641-646. https://doi. org/10.1016/S0361-9230(97)00286-4

Nevo E (1999) Mosaic evolution of subterranean mammals. Oxford University Press, Oxford

Nevo E, Heth G, Beiles A, Frankenberg E (1987) Geographic dialects in blind mole-rats: role of vocal communication in active speciation. Proc Natl Acad Sci 84:3312-3315. https://doi.org/10.1073/ pnas.84.10.3312

Pepper JW, Braude SH, Lacey EA, Sherman PW (1991) Vocalizations of the naked mole-rat. In: Sherman PW, Jarvis JUM, Alexander RD (eds) The biology of the naked mole-rat. Princeton University Press, Princeton, pp 243-274

Picton TW, Woods DL, Baribeau-Braun J, Healey TM (1976) J Otolaryngol 6:90-119
Robier TC, Fabry DA, Leek MR, Van Summers W (1992) Improving the frequency specificity of the auditory brain stem response. Ear Hear 13:223-227. https://doi.org/10.3109/01050398609070689

Ryan A (1976) Hearing sensitivity of the Mongolian gerbil, Meriones unguiculatus. J Acoust Soc Am 59:1222-1226. https://doi. org/10.1121/1.380961

Ryan A (1986) Factors influencing the evolution of acoustic communication: biological constraints. Brain Behav Evol 28:70-82

Sisneros JA, Bass AH (2003) Seasonal plasticity of peripheral auditory frequency sensitivity. J Neurosci 23:1049-1058. https://doi. org/10.1523/JNEUROSCI.23-03-01049.2003

Starr A, Achor J (1975) Auditory brain stem responses in neurological disease. Arch Neurol 32:761-768. https://doi.org/10.1001/archn eur.1975.00490530083009

Veitl S, Begall S, Burda H (2000) Ecological determinants of vocalization parameters: the case of the coruro Spalacopus cyanus (Octodontidae), a fossorial social rodent. Bioacoustics 11:129-148. https://doi.org/10.1080/09524622.2000.9753455

Werner LA, Folsom RC, Mancl LR (1993) The relationship between auditory brainstem response and behavioral thresholds in normal hearing infants and adults. Hear Res 68:131-141. https://doi. org/10.1016/0378-5955(93)90071-8

Woolley S, Moore JM (2011) Coevolution in communication senders and receivers: vocal behavioral and auditory processing in multiple songbird species. Ann NY Acad Sci 1125:155-165. https:// doi.org/10.1111/j.1749-6632.2011.05989.x

Wright TF, Brittan-Powell EF, Dooling RJ, Mundinger PC (2004) Sexlinked inheritance of hearing and song in the Belgian Waterslager canary. Proc Biol Sci 6:S409-S412. https://doi.org/10.1098/ rsbl.2004.0204

Yosida S, Okanoya K (2009) Naked mole-rat is sensitive to social hierarchy encoded in antiphonal vocalization. Ethology 115:823-831. https://doi.org/10.1111/j.1439-0310.2009.01677.x

Yosida S, Kobayasi KI, Ikebuchi M, Ozaki R, Okanoya K (2007) Antiphonal vocalization of a subterranean rodent, the naked molerat (Heterocephalus glaber). Ethology 113:703-710. https://doi. org/10.1111/j.1439-0310.2007.01371.x 